\title{
Editorial
}

\section{National Nutrition Month 2021: nutrition for protection}

\author{
Anil Samaranayake \\ Director, Nutrition Division, Ministry of Health, Sri Lanka \\ Correspondence: nutritiondivision@health.gov.lk \\ https://orcid.org/0000-0002-0212-2533 \\ DOI: https://doi.org/10.4038/jccpsl.v27i4.8478
}

Received on: 16 December 2021

Accepted on: 30 December 2021

\begin{abstract}
A healthy balanced diet includes a variety of healthy food in correct amounts from identified food groups as per the recommendations. It provides all nutrients necessary to protect against malnutrition in all its forms, as well as preventing and controlling noncommunicable diseases (NCD).
\end{abstract}

Achieving proper nutrition through healthy eating is important during this COVID-19 pandemic era as healthy balanced diets help to improve immunity of an individual. The human immune system is influenced by a balance of many factors especially a healthy lifestyle including consumption of a balanced diet consisting of macronutrients (carbohydrates, proteins, and fats), a range of micronutrients (e.g., vitamins and minerals), and non-nutritive compounds (e.g., phytochemical including antioxidants) combined with low stress, adequate sleep and exercise.

Sri Lanka first published the Food-Based Dietary Guidelines (FBDG) in 2002. A revised version was launched in 2011. In 2020, Nutrition Division of the Ministry of Health undertook the task of reviewing and updating the guidelines, in view of incorporating the evidence-based latest information along with the global concept of the environmentally sustainable diet. Hence, the community is given timely and appropriate guidance on healthy diet and lifestyle that is persistently subjected to required shifts.
The new version of FBDG includes comprehensive knowledge on general and specific recommendations which the healthcare workers will use to direct the community on healthy food choices and correct dietary practices that will lead to the ultimate goal of a positive behavioural change which will result in optimum dietary diversification. Undoubtedly, FBDG s developed by the Nutrition Division of the Ministry of Health will sensitize the public on healthy diet and will contribute towards achieving best nutrition standards for all.

The FBDGs provide evidence-based guidance to serve as a tool for promoting healthy eating which helps prevention of diseases and achieve overall wellbeing. There are 14 key general guidelines and four age-specific guidelines based on food recommended for Sri Lankans, which will be available to the health workers and public in all three languages.

- Add colour to your daily meals balancing the correct amounts

The essential food that makes up a healthy dietary pattern include cereals and starchy foods, vegetables and green leaves, fruits, pulses, animal sources of protein (e.g., fish, sea food, eggs, lean meats), fresh milk and its fermented products, nuts and oily seeds. Some of these foods together with condiments and herbs have functional 
properties providing health benefits beyond their nutritional value.

- Eat whole grains and their products including less polished or parboiled rice instead of refined grains and their products

Whole grains and parboiled rice add vitamins and minerals to our diet which is lacking in refined grains and their products. Except for rice, other commonly used cereals in Sri Lanka are kurakkan, maize and wheat.

- Eat at least two vegetables, one green leafy vegetable and two fruits daily

Sri Lanka is blessed with a wide variety of fruits and vegetables throughout the year, while some are seasonal or locally available. Each fruit or vegetable is different in taste, colour and texture and add variety to our diet. They are rich in vitamins, minerals, antioxidants and fibre that help to improve immune response and prevent and control NCDs. Fruits and vegetables can be consumed as a healthy snack while some of them have medicinal properties (e.g., bitter gourd, thebu). The consumption of at least $400 \mathrm{~g}$ of edible vegetables, green leaves and fruits daily provides vitamins, minerals and fibre in required amounts. It is the same when consuming two vegetables and one green leaves ( 3 tablespoons each) and two fruits a day.

- Eat fish or egg or lean meat with pulses at every meal

Pulses, fish, eggs, poultry and lean meat are major sources of protein in our diet. These foods are also rich in vitamins and minerals. Animal sources of proteins are good quality proteins as they contain all essential amino acids which cannot be synthesized in the body. Pulses are better absorbed when consumed with cereals. At the same time, plant sources of protein support environmentally sustainable healthy diet and do not cause heart diseases.
- Limit sugary drinks, biscuits, cakes, sweets and sweeteners

Sugar is addictive and sugar (table sugar/sucrose) that we add to food and beverages provides empty calories which do not have other nutrients except for carbohydrates. Consumption of sugarsweetened beverages (SSB), biscuits, cakes and other sweets promotes excess energy intake leading to inflammatory diseases, obesity and other NCDs.

\section{- Have fresh milk and its fermented products}

Fresh milk and its fermented products are not necessary to be a part of daily diet. However, they can contribute to dietary diversity when taken on individual preference within recommended amounts. Limit consumption of sweetened, coloured and flavoured milk, yogurts, yoghurt drinks, and condensed milk.

- Eat a handful of nuts or oily seeds daily

Everyone needs moderate amounts of fat in the diet. Daily requirement of unsaturated fat can be met by consumption of cashew nuts, peanuts, kottang, pumpkin seeds, gingelly (sesame) and avocado. Eating a handful (on the average $30 \mathrm{~g}$ ) of nuts and oily seeds is helpful to achieve the required amounts of monounsaturated fats, which are the healthier options of fat.

\section{- Limit salty food and adding salt to food}

The current salt consumption exceeds the recommended amount of less than one teaspoon of iodized salt per person per day. Salt taste is addictive and has negative health implications. Adding salt to food at the table and rice when cooking should be avoided. Herbs, spices, garlic and lime/lemon juice can be used to enhance the taste instead of salt and artificial flavour enhancers (e.g., mono sodium glutamate (MSG), seasoning cubes) which are high in sodium. 
- Drink 8-10 glasses (1.5-2.01) of water throughout the day

Water should be the beverage of choice and it is calorie-free. It plays an integral role in many vital functions in our body. Drinking water should be clean and safe. Other than water, fluids such as king coconut water, herbal drinks, tea and fresh fruit juice without added sugar also help to ensure daily hydration.

- Be active: engage in moderate physical activity for at least 150-300 minutes per week

Regular physical activity improves overall health and reduces the risk of many health issues at all ages. Doing some physical activity is better than none and more is better. All types of physical activities count; they can be done as part of work, sport, leisure or transport.

\section{- Sleep 7-8 hours continuously everyday}

Sleep is a state where the body reduces the awareness to environmental stimuli, ensuring both physical and mental wellbeing. Optimize your sleep schedule and daily routines for a quality sleep. Avoid sleep soon after having a meal and switch-off digital screens at least an hour before sleep. Avoid alcohol, smoking and caffeinated drinks in the evening.

\section{- Eatclean andsafefood}

Food must be clean and safe for consumption. Food safety must be considered during selection, storage and preparation of food in order to avoid contamination and its harmful health consequences. Growing fruits and vegetables organically in a home garden is encouraged as much as possible.
It is best to avoid storing cooked food at room temperature for more than two hours as spoilage may begin. Do not store cooked food too long even in the refrigerator.

- Eat fresh and home cooked food: limit processed and ultra-processed foods

Home-cooked meals can be prepared in a healthy manner using fresh produce, with less salt, sugar and unhealthy fats (saturated or trans fats) and be prepared in different ways according to preferences (e.g., curries, soups and broths, preserves, salads, 'mellum', pickles, drinks, desserts).

Prepare food with natural culinary ingredients (e.g., herb, spices, lime juice) and avoid food additives as much as possible.

- Always read labels of packaged food and beverages

If buying packaged food, always read the label for ingredients, nutrient values, date of expiry and instructions for storage and preparation. Follow the colour coding system for fat, salt and sugar in solid and semi-solid food. Check amounts of desired nutrients or types of additives and levels of fat, salt, and sugar in food labels to identify hidden unhealthy contents.

A diversified, balanced and healthy diet may vary depending on individual characteristics such as age, gender, place of residence, level of physical activity, cultural context and customs, and locally available foods. However, the basic principles of healthy eating remain the same and should follow the dietary recommendations. 